\title{
Temporal patterns of schedule-induced drinking and pawgrooming in rats exposed to periodic food
}

\author{
CINDY P. LAWLER and PERRIN S. COHEN \\ Northeastern University, Boston, Massachusetts
}

\begin{abstract}
Five rats were exposed to fixed-time food schedules, ranging from 30 to $480 \mathrm{sec}$. Three rats displayed a postfood pattern of schedule-induced drinking, with short latencies from food delivery to drinking at all interfood interval durations. In contrast, drinking for the other 2 subjects tended to occur at lower overall levels, and drinking bouts frequently began in the middle of the interfood interval, such that the latency from food delivery to drinking increased dramatically as the interfood interval increased. Observation of these 2 subjects revealed that another form of licking-pawgrooming - occurred reliably after food delivery and before drinking bouts. A betweensubject comparison of the 3 postfood drinkers and the 2 pawgroomers revealed that, in addition to a common topography (repetitive licking), pawgrooming and drinking were similar with respect to their temporal locus, relation to the interfood interval, and extinction baseline levels. These similarities suggest that drinking and pawgrooming are induced by a common mechanism. Cohen, Looney, Campagnoni, and Lawler's (1985) two-state model of reinforcer-induced motivation provides a useful framework for the interpretation of these results.
\end{abstract}

When hungry rats receive small portions of food intermittently, distinctive behaviors emerge in the period following each food delivery. These behaviors include excessive drinking, chewing and ingesting nonfood substances (pica), attack, general locomotor activity, and responding to change the stimuli associated with the food schedule (time-out responding) (Falk, 1971, 1981; Killeen, 1975; Staddon, 1977). Falk (1971) coined the term adjunctive to call attention to similarities among these behaviors, and to distinguish them from instances of classical or operant conditioning. The temporal locus of adjunctive behaviors has been particularly important to speculations that they reflect an arousing (Cantor, 1981; Cook, Wallace, \& Singer, 1983; Killeen, 1975; Wayner, 1970 ) or aversive (Azrin, Hutchinson, \& Hake, 1966; Rosellini, 1985; Staddon, 1977) aftereffect of the prior food delivery.

\footnotetext{
We are especially grateful to Harry Mackay, Adam Reeves, and Jim Stellar for their careful review and helpful suggestions on early drafts of the manuscript. Clorinda Creo, Theodore Kozlowski, Rozana Troiano, Monique Antoine, and Daniel Tamkin provided invaluable help with running the experiment. Kathy Quist and Mark Krietz devoted many hours to learning the observational scoring system and establishing interrater reliability. This research was presented to the Graduate School of Arts and Sciences of Northeastern University by Cindy P. Lawler in partial fulfillment of the requirements for the doctor of philosophy degree. Preliminary data from this experiment were presented in March 1985 at the annual meeting of the Eastern Psychological Association in Boston, Massachusetts. The research was supported in part by OUSTS Grant RR07413 to Northeastern University. Requests for reprints shouid be addressed to Cindy P. Lawler, Room 304, Brain and Development Research Center, CB $\# 7250$, University of North Carolina, Chapel Hill, NC 27599-7250.
}

Cohen, Looney, Campagnoni, and Lawler (1985) and Campagnoni, Lawler, and Cohen (1986) reported interesting differences in the temporal properties of several schedule-induced (adjunctive) behaviors in pigeons. They found that attack is reactive, occurring immediately postfood and reaching peak levels at the same absolute time (5-10 sec after food delivery), regardless of the overall length of the interfood interval. In contrast, three other induced behaviors, increased general activity, time-out responding, and movement away from the food site, occur after attack and exhibit scalar (proportional) timing (Gibbon, 1977). In other words, the onset of these activities depends on the overall interval between food deliveries, that is, they reach peak levels at the same relative point $(25 \%-35 \%$ of the interfood interval) after food.

Cohen et al. (1985) have proposed a two-state model of reinforcer-induced motivation as a way of interpreting these results. The model states that differences in the temporal characteristics of schedule-induced behaviors reflect the operation of two independent motivational states (State I and State II). Aggression in pigeons is a prototypical State I behavior. General activity, time-out responding, and movement away from the food site are examples of State II behaviors. A crucial tenet of the model is that State I behaviors are independent of, and thus do not compete with, State II behaviors so that, for example, removing the opportunity for either aggression or drinking (presumed State I behaviors) does not alter the level or temporal pattern of time-out responding, a State II behavior (Ator, 1980; Brantley, 1977).

In addition to independent sources of temporal control, the behaviors associated with the two states are distin- 
guished topographically and functionally. State I induces repetitive, stereotypical, species-specific activities that may play a role in modulating physiological stress during exposure to intermittent schedules of food availability (e.g., Brett \& Levine, 1979, 1981; Mittleman, Jones, \& Robbins, 1988). In contrast, State II potentiates gross skeletal and locomotor activities that represent an induced tendency to explore the environment (Berlyne, 1960). From this perspective, time-out responding reflects the positively reinforcing value of stimulus change rather than a tendency to escape from the reinforcer schedule (see Cohen \& Campagnoni, 1989).

Is the prototypical adjunctive behavior-drinking-a State I or State II activity? Similar to attack in pigeons, induced drinking in rats is a highly stereotyped, speciesspecific activity, typically reported to occur soon after food delivery (J. D. Allen, personal communication, July 1983; Lucas, Timberlake, \& Gawley, 1988; Roper, 1978; Shurtleff, Delamater, \& Riley, 1983) and before activities such as wheel running and time-out responding (Brantley, 1977; Segal, 1969a; Staddon \& Ayers, 1975; Wetherington \& Riley, 1986). These data support Cohen et al.'s (1985) contention that drinking is a reactive State I process. Such a conclusion is tentative, however, because most studies that report the temporal distribution of induced drinking provide poor temporal resolution or are restricted to interfood intervals of $2 \mathrm{~min}$ or less. To add to the uncertainty, the temporal data from two studies that examined long interfood intervals (greater than $2 \mathrm{~min}$ ) (Rosellini \& Burdette, 1980; Segal, Oden, \& Deadwyler, 1965) report that drinking may appear at times other than the immediate postfood period.

In view of the inconclusive data, we conducted a parametric within-subject examination of the temporal distribution of induced drinking during periodic fixed-time (FT) food schedules with a wide range of interfood interval values. Our primary purpose was to determine whether drinking showed the expected State I pattern (i.e., occurred shortly after each food delivery, at the same absolute time in the interfood interval during each condition) or a State II pattern (i.e., occurred late in the postfood period, at the same relative time in the interfood interval).

Once the experiment was under way, we noticed that 2 subjects engaged in an unexpected topography (repetitive pawgrooming, i.e., licking and biting of the forepaws) during the postfood period. We systematically measured repetitive pawgrooming for these 2 subjects during the second half of the experiment with the intent to document a previously unreported adjunctive behavior and to compare its characteristics with those of induced drinking.

\section{METHOD}

\section{Subjects}

Five experimentally naive adult male albino (Sprague-Dawley) rats, obtained from Charles River Breeding Laboratories (Arlington, MA), served as subjects. The rats were housed individually in clear polycarbonate cages. Each rat was reduced to $80 \%$ of its free-feeding baseline; this weight was maintained throughout the experiment. Water was available freely in the home cages. The colony room operated on a $14: 10-\mathrm{h}$ light:dark cycle. Sessions were conducted during the light phase.

\section{Apparatus}

Four identical rodent-conditioning chambers (Gerbrands, Model C) were used. Each chamber measured $19.3 \times 23.4 \times$ $20.3 \mathrm{~cm}$. A recessed food tray was attached behind an opening in the front chamber wall. An infrared photo emitter and detector sensed food-tray entries. A water bottle was mounted outside the left side wall of the chamber, with the spout protruding $0.6 \mathrm{~cm}$ through an opening $4 \mathrm{~cm}$ above the chamber floor. The shortest distance between the spout and the food tray was $13.6 \mathrm{~cm}$. A Gerbrands contact sensor (Model G6350P) detected licks at the spout. A TRS-80 Model III microcomputer controlled experimental events and sampled drinking and food-tray entries 10 times/sec.

\section{Procedure}

Sessions were conducted six times per week. After 1 day of magazine training, each subject was exposed to 25 sessions of a fixedtime (FT) 120-sec schedule. Following the development of induced drinking at FT $120 \mathrm{sec}$, each rat was exposed to a logarithmic series of FT schedules, ranging from 30 to $480 \mathrm{sec}$ (see Table 1). The sequence of FT schedules began with a partial descending series $(120-30 \mathrm{sec})$, followed by a complete ascending series $(30-480 \mathrm{sec})$. The last phase of the experiment was a complete descending series $(480-30 \mathrm{sec})$ of FT schedules. Each food delivery consisted of a 45-mg Noyes food pellet (traditional Formula A). Food pellets were delivered at fixed times, independently of a subject's behavior.

Each session began and ended with a food delivery, and consisted of 60 interfood intervals, except that at the longest interval value (FT $480 \mathrm{sec}$ ), only 31 food pellets were delivered. Each condition was in effect for at least 15 sessions ( 30 sessions for FT $480 \mathrm{sec}$ ), or until water intake and the temporal distributions of food-tray entry and drinking appeared stable; that is, variability across five successive sessions for a subject was neither decreasing nor increasing. One baseline extinction session was programmed at the end of each condition. During this session, the pellet chute was disconnected from the food tray; the dispenser continued to click at the end of each programmed interval, yet no pellets were delivered. Table 1 summarizes the sequence of experimental conditions and number of sessions for each rat.

Changeover delay. To avoid unduly constraining the temporal distributions of drinking, no changeover delay was initially programmed between licking and food-pellet delivery. An exception occurred for Subject CP5, however. During the second exposure to FT $240 \mathrm{sec}$ (Condition 8), the distribution of drinking changed dramatically for this subject; instead of occurring in the early or middle portion of each interval, drinking began to increase gradually throughout the interfood interval, reaching a peak in the terminal (Staddon \& Simmelhag, 1971) period (immediately before the next food delivery). This pattern is typically associated with behaviors directed toward the scheduled reinforcer. A similar sudden and dramatic change from a postfood to a prefood temporal locus has been previously reported for both induced drinking (Segal, 1969b) and attack (Campagnoni et al., 1986). To discourage the potential development of drinking as a terminal behavior in the present case, a changeover delay (COD) was programmed between drinking and food-pellet delivery for CP5. Within a few sessions, drinking returned to the early and middle portions of the interfood interval. For this subject, a COD of 10-20 sec (see Table 1) was in effect for the remainder of the experiment. An examination of the records at the end of the experiment revealed that in the two conditions immediately following the institution of the COD (FT 240 and FT $120 \mathrm{sec}$; Conditions 8 and 9), CP5 came into contact with this contingency infrequently. There were occasional instances in 
Table 1

Number of Sessions and Sequence of Experimental Conditions

\begin{tabular}{|c|c|c|c|}
\hline Subject & Condition & $\begin{array}{c}\text { Fixed-Time } \\
\text { Schedule (sec) }\end{array}$ & $\begin{array}{c}\text { No. of } \\
\text { Sessions }\end{array}$ \\
\hline CPI & $\begin{array}{c}1 \\
2 \\
3 \\
4 \\
5 \\
6^{*} \\
7^{*} \\
8^{*} \\
9^{*} \\
10^{*} \\
11^{*}\end{array}$ & $\begin{array}{r}120 \\
60 \\
30 \\
60 \\
120 \\
240 \\
480 \\
240 \\
120 \\
60 \\
30\end{array}$ & $\begin{array}{l}26 \\
18 \\
16 \\
18 \\
18 \\
28 \\
39 \\
16 \\
17 \\
21 \\
36\end{array}$ \\
\hline $\mathrm{CP} 2$ & $\begin{array}{r}1 \\
2 \\
3 \\
4 \\
5 \\
6 \\
7 \\
8 \\
9 \\
10 \\
11\end{array}$ & $\begin{array}{r}120 \\
60 \\
30 \\
60 \\
120 \\
240 \\
480 \\
240 \\
120 \\
60 \\
30\end{array}$ & $\begin{array}{l}26 \\
16 \\
17 \\
17 \\
17 \\
28 \\
31 \\
21 \\
30 \\
31 \\
33\end{array}$ \\
\hline $\mathrm{CP} 3$ & $\begin{array}{r}1 \\
2 \\
3 \\
4 \\
5 \\
6 \\
7 \\
8 \\
9 \\
10 \\
11\end{array}$ & $\begin{array}{r}120 \\
60 \\
30 \\
60 \\
120 \\
240 \\
480 \\
240 \\
120 \\
60 \\
30\end{array}$ & $\begin{array}{l}27 \\
16 \\
17 \\
19 \\
16 \\
24 \\
31 \\
32 \\
19 \\
23 \\
22\end{array}$ \\
\hline CP4 & $\begin{array}{r}1 \\
2 \\
3 \\
4 \\
5 \\
6 \\
7 \\
8 \\
9 \\
10 \\
11\end{array}$ & $\begin{array}{r}120 \\
60 \\
30 \\
60 \\
120 \\
240 \\
480 \\
240 \\
120 \\
60 \\
30\end{array}$ & $\begin{array}{l}27 \\
17 \\
17 \\
19 \\
16 \\
22 \\
32 \\
17 \\
16 \\
20 \\
17\end{array}$ \\
\hline CP5 & $\begin{array}{c}1 \\
2 \\
3 \\
4 \\
5^{*} \\
6^{*} \\
7^{*} \\
8^{*} \\
9^{*} \\
10^{*} \\
11^{*}\end{array}$ & $\begin{array}{c}120 \\
60 \\
30 \\
60 \\
120 \\
240 \\
480 \\
240 \dagger \\
120 \dagger \\
60 \ddagger \\
30 \$\end{array}$ & $\begin{array}{l}26 \\
16 \\
17 \\
20 \\
28 \\
21 \\
31 \\
26 \\
20 \\
31 \\
17\end{array}$ \\
\hline
\end{tabular}

*Conditions where pawgrooming was observed and recorded formally for CP1 and CP5. $\quad \nmid 20-\mathrm{sec}$ changeover delay (COD). $\quad \ddagger 15$-sec COD. $\$ 10$-sec COD.

the last two conditions of the experiment (FT $60 \mathrm{sec}$ and FT $30 \mathrm{sec}$; Conditions 10 and 11 ), however, when the end of a long drinking bout extended into the last third of the interfood interval and caused slight increases in the programmed interfood interval.

Observation of repetitive pawgrooming. We noticed the occurrence of repetitive pawgrooming for both CP1 and CP5 during brief informal observations of selected sessions within the first half of the experiment. The development of pawgrooming for CP5 did not, however, coincide with the brief appearance of prefood drinking during the second FT $240-\sec$ condition. During the second half of the experiment, we systematically observed and recorded pawgrooming for both CPI and CP5. Such pawgrooming was defined as perseverative licking and biting of the forepaws. During a bout of pawgrooming, the forepaws remained in close contact with the mouth, and only small rhythmical movements of the paws under the mouth occurred. This category of behavior excluded all other forms of grooming, including face grooming (large sweeping movements of the paws over the nose, eyes, and ears) as well as body grooming (licking, biting, and scratching other parts of the body). Our observations of CP1 and CP5 indicated a clear distinction between isolated bouts of repetitive pawgrooming (the response of interest) and brief pawgrooming episodes that accompanied other grooming activities. Brief (less than $2 \mathrm{sec}$ ) pawgrooming bouts that were immediately (within $2 \mathrm{sec}$ ) followed or preceded by other forms of grooming were excluded from analysis; thus, the pawgrooming category was reserved for isolated bouts of repetitive licking and biting of the forepaws.

Our initial analysis of pawgrooming during several successive sessions indicated high across-session stability; therefore, formal observation of CP1 and CP5 was limited to a single session (within the final six sessions) during each FT schedule. A second analysis of across-session stability during the final six sessions for both of the last two conditions of the experiment (Conditions 10 and 11 ) confirmed that there was small intersession variability in measures of pawgrooming. During the longer FT schedules (240 and $480 \mathrm{sec}$ ), sessions lasted $2 \mathrm{~h}$. In these cases, observation was accomplished over $\mathbf{2}$ or more days, with pawgrooming measured in different portions of successive daily sessions. Pawgrooming was also measured during extinction sessions. The same observer was used throughout the experiment. The beginning and end of a pawgrooming bout were recorded by entering codes into a TRS- 80 Model III microcomputer. To assess interobserver reliability, another individual was trained to score pawgrooming; this individual was naive as to the purpose and expected results of the experiment. Interobserver reliability was measured during two sessions of the FT 60-sec schedule (Condition 10) and two sessions at the FT 30-sec schedule (Condition 11); the Pearson product-moment correlation coefficient $(r)$ between observers was 0.98 .

\section{RESULTS}

Except where indicated, the data presented are means and their standard errors for the last 5 days of each condition.

\section{Baseline Levels of Drinking}

To determine whether drinking was induced by the fixed-time food schedules (Cohen \& Looney, 1984) we compared the amount of water ingested (in milliliters) during each condition with the amount ingested during the corresponding extinction baseline. With one exception (CP1, FT $480 \mathrm{sec}$; Condition 7), water intake was at least doubled during extinction baseline sessions (Table 2).

\section{Temporal Patterns of Drinking}

We examined the temporal pattern of drinking in the interval between food deliveries to determine whether drinking followed a State I pattern (i.e., occurred immediately after food delivery, at the same absolute time in the interfood interval) or a State II pattern (i.e., occurred late 
Table 2

Means and Standard Errors of Session Water Intake (in Milliliters) for Each Subject and Condition

\begin{tabular}{|c|c|c|c|c|c|c|c|c|c|c|c|c|c|c|c|c|}
\hline \multirow[b]{3}{*}{ Condition } & \multirow[b]{3}{*}{ FT (sec) } & \multicolumn{15}{|c|}{ Subjects } \\
\hline & & \multicolumn{3}{|c|}{ CPI } & \multicolumn{3}{|c|}{$\mathrm{CP} 2$} & \multicolumn{3}{|c|}{ CP3 } & \multicolumn{3}{|c|}{ CP4 } & \multicolumn{3}{|c|}{ CP5 } \\
\hline & & $M$ & $S E$ & $\mathrm{EXT}^{*}$ & $M$ & $S E$ & EXT & $M$ & $S E$ & EXT & $M$ & $S E$ & EXT & $M$ & $S E$ & EXT \\
\hline 1 & 120 & 14.6 & 1.8 & 4.0 & 42.8 & 4.6 & 2.0 & 24.5 & 1.4 & 5.0 & 47.4 & 3.5 & 6.0 & 26.6 & 2.6 & 6.0 \\
\hline 2 & 60 & 15.1 & 1.2 & 1.0 & 35.0 & 1.8 & 10.0 & 29.3 & 1.3 & 3.5 & 43.6 & 2.5 & 15.0 & 22.4 & 1.3 & 1.0 \\
\hline 3 & 30 & 22.4 & 1.7 & 1.0 & 21.4 & 1.6 & 2.0 & 28.0 & 2.5 & 2.0 & 33.2 & 1.6 & 1.0 & 22.8 & 1.5 & 7.0 \\
\hline 4 & 60 & 24.2 & 1.2 & 2.0 & 32.2 & 2.4 & 2.5 & 40.2 & 1.4 & 2.0 & 39.8 & 0.9 & 0.0 & 28.0 & 1.5 & 2.0 \\
\hline 5 & 120 & 17.2 & 2.1 & 0.0 & 36.2 & 1.1 & 7.0 & 33.2 & 2.9 & 1.0 & 41.2 & 1.0 & 0.0 & 23.0 & 1.3 & 10.0 \\
\hline 6 & 240 & 12.0 & 2.9 & 2.0 & 29.4 & 1.0 & 4.0 & 19.2 & 2.4 & 1.0 & 36.6 & 1.3 & 0.0 & 24.6 & 3.7 & 6.0 \\
\hline 7 & $480 \dagger$ & 3.3 & 1.4 & 3.0 & 4.4 & 0.8 & 0.0 & 4.8 & 0.8 & 1.0 & 14.5 & 1.1 & 0.0 & 7.0 & 1.8 & 2.0 \\
\hline 8 & 240 & 11.0 & 1.2 & 5.0 & 9.0 & 1.1 & 4.0 & 8.2 & 1.0 & 3.0 & 29.6 & 2.1 & 1.0 & 14.2 & 1.5 & 6.0 \\
\hline 9 & 120 & 14.6 & 0.5 & 0.0 & 29.8 & 1.4 & 8.0 & 22.4 & 2.3 & 8.0 & 30.4 & 1.2 & 1.0 & 31.2 & 1.5 & 4.0 \\
\hline 10 & 60 & 15.0 & 0.5 & 3.0 & 24.6 & 1.5 & 3.0 & 25.6 & 2.9 & 2.0 & 31.0 & 1.1 & 1.0 & 16.6 & 0.7 & 0.0 \\
\hline 11 & 30 & 16.8 & 1.0 & 0.5 & 19.8 & 2.0 & 2.0 & 19.8 & 1.5 & 5.0 & 27.0 & 1.3 & 1.0 & 14.2 & 0.5 & 4.0 \\
\hline
\end{tabular}

*Intake shown for each extinction condition (EXT) represents the total intake during a single session. fOnly 31 reinforcers were delivered per session during the fixed-time (FT) 480-sec condition. All other conditions consisted of sessions with 61 reinforcers.

in the postfood period, at the same relative time in the interfood interval).

Drink latency. Figure 1 shows the median latency to drink following a pellet delivery for each subject as a function of the interfood interval duration. Median latency, rather than mean latency, was chosen as the most appropriate measure, due to the potential confounding of mean latency measures by occasional spurious contacts between the subject and the water spout. Such spurious contacts would be expected to differentially bias latency measures at long interfood intervals because there was more total time available for spurious contacts in those conditions, and also because the reduced overall drink frequency at long interfood intervals increased the probability that spurious contacts occurring late in the interfood interval would be recorded as the first drink to occur. The

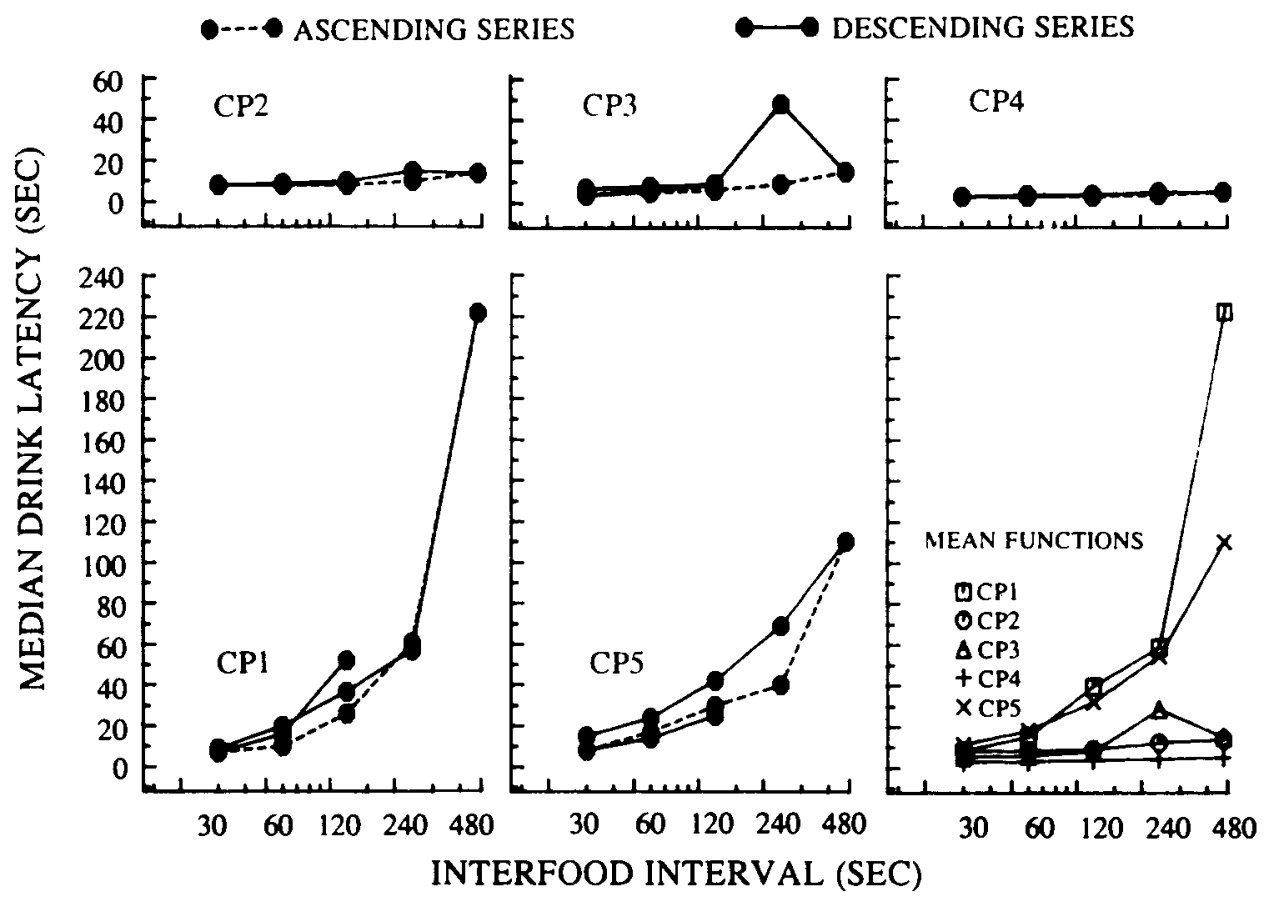

Figure 1. Median time (in seconds) from pellet delivery to onset of drinking for each subject, and interfood interval duration. The abscissa is plotted in logarithmic coordinates to allow compact presentation of the data. Three separate functions are plotted on each graph. The two solid lines represent the partial (Conditions 1-2, Table 1) and complete (Conditions 8-11, Table 1) descending series of FT schedules. The dashed line represents data from the complete ascending series (Conditions 3-7, Table 1) of FT schedules. To make across-subject comparisons easier, each function plotted in the graph in the lower right corner represents the means of the ascending and descending functions for an individual subject. 
median latency data reveal interesting differences between subjects. For Subjects CP2, CP3, and CP4, the median latency to drink increased slightly as the overall interval length increased. The one exception was a large increase in latency at FT $240 \mathrm{sec}$ during the descending series for CP3. If this single large increase is disregarded, the change in latency from the shortest to the longest interfood interval was 7, 8, and 2 sec for CP2, CP3, and CP4, respectively. In contrast to these small increases, drink latency for CP1 and CP5 increased dramatically, from 8 to $222 \mathrm{sec}$ for CP1, and from 12 to $110 \mathrm{sec}$ for CP5, over the same range of interfood intervals.

The drink latency data displayed in Figure 1 raised the possibility that drinking for 2 subjects (CP1 and CP5) fit a State II pattern, such that drink latency increased in proportion to the increase in interfood interval. To explore this possibility, the latency data for these 2 subjects were replotted in logarithmic coordinates for both ordinate and abscissa. The transformed data are depicted in Figure 2 (top panel). A proportional increase in drink latency would yield a linear function with a slope of unity. Such a slope would be consistent with a State II pattern. In contrast,

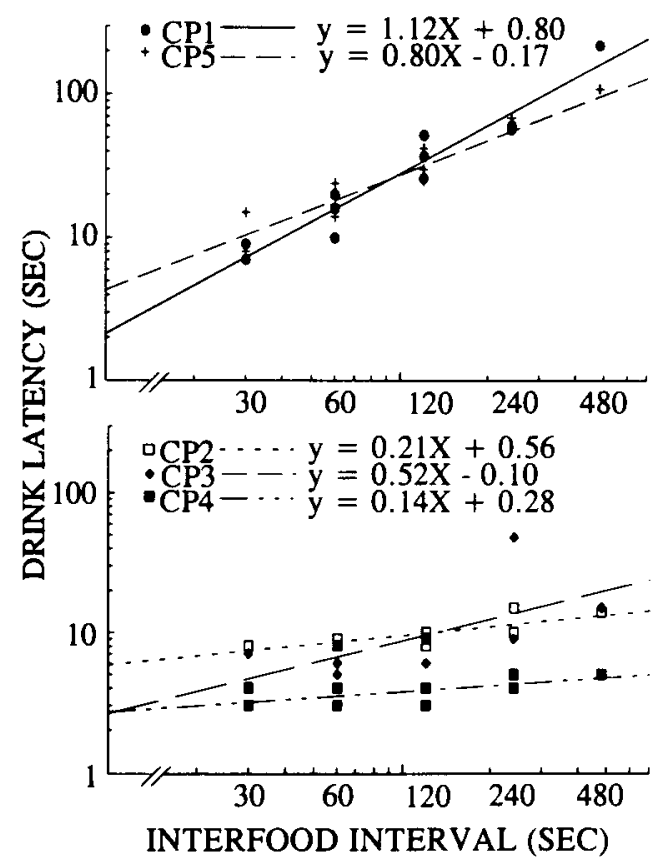

Figure 2. Regression analysis of time (in seconds) from pellet delivery to onset of drinking for CP1 and CP5 (upper panel) and CP2, CP3, and CP4 (lower panel). Both the abscissa and the ordinate are plotted in logarithmic coordinates. The functions displayed are regression lines derived from a least squares linear correlation analysis of the plotted data ( $\log _{10}$ drink latency vs. $\log _{10}$ interfood interval). Linear equations shown are of the form $y=(m \cdot X)+b(m=$ slope; $b=y$-intercept), and correspond to the plotted regression lines. Regression coefficients $(r)$ are 0.96 (CP1); 0.82 (CP2); 0.68 (CP3); 0.65 (CP4); and 0.94 (CP5). a slope near zero would be obtained if drink latency were constant, as expected for State I behaviors. A linear least squares regression analysis was performed on the transformed data; the resulting regression lines are shown in Figure 2. The slope of the best-fit line was 1.12 for CP1 and 0.8 for CP5. In contrast, a regression analysis of the data for the remaining 3 subjects yielded much lower slopes; $0.21,0.52$, and 0.14 for CP2, CP3, and CP4, respectively (see lower panel in Figure 2).

Distribution of drinking within the interfood interval. The between-subject differences depicted in Figures 1 and 2 are also reflected in Figures 3 and 4, which present the probability of drinking for each second within the interfood interval for each subject. Three subjects (CP2, CP3, and CP4) displayed a postfood drinking pattern. That is, in almost all cases, distributions of drinking were skewed negatively and reached a maximum within the first $20 \mathrm{sec}$ of the interval. At the longer interval values, the peak probability of drinking was reduced. In those cases, drinking also tended to extend further into the interval, so that the distributions became progressively flatter. There was a slight but systematic tendency for drinking distributions to shift to the right (toward later postfood times) as the interval length was increased.

In contrast to the postfood drinking displayed by $\mathrm{CP} 2$, $\mathrm{CP} 3$, and CP4, the remaining 2 subjects (CP1 and CP5) displayed a midinterval drinking pattern. As seen in Figures 3 and 4, drinking distributions for CP1 and CP5 were flatter and more normally distributed within the interval, with the exception of the shorter intervals. As the interval value increased, peak levels of drinking showed large shifts toward later times in the interfood interval, such that drinking typically occurred in the middle of the interval. Compared to the other 3 subjects, the increased variability at longer interval lengths was more pronounced.

To determine whether the drinking distributions for CP1 and CP5 occurred at the same relative point in the interfood interval (as expected for State II behaviors), the drinking distributions for these 2 subjects were replotted as a function of proportion of the interfood interval in Figure 5. Under these conditions, State II behaviors would be expected to show largely overlapping distributions. In general, the distributions for both CP1 and CP5 displayed greater overlap when plotted as a function of proportion of the interfood interval (in Figure 5) than when plotted as a function of absolute time since food (in Figures 3 and 4).

\section{Frequency of Drinking}

We examined the number of interfood intervals containing drinking to determine whether the between-subject differences in drinking distributions were accompanied by differences in the frequency of drinking. Figure 6 shows the percentage of interfood intervals with drinking for each subject and condition. In general, the percentage of intervals with drinking decreased as the interfood 


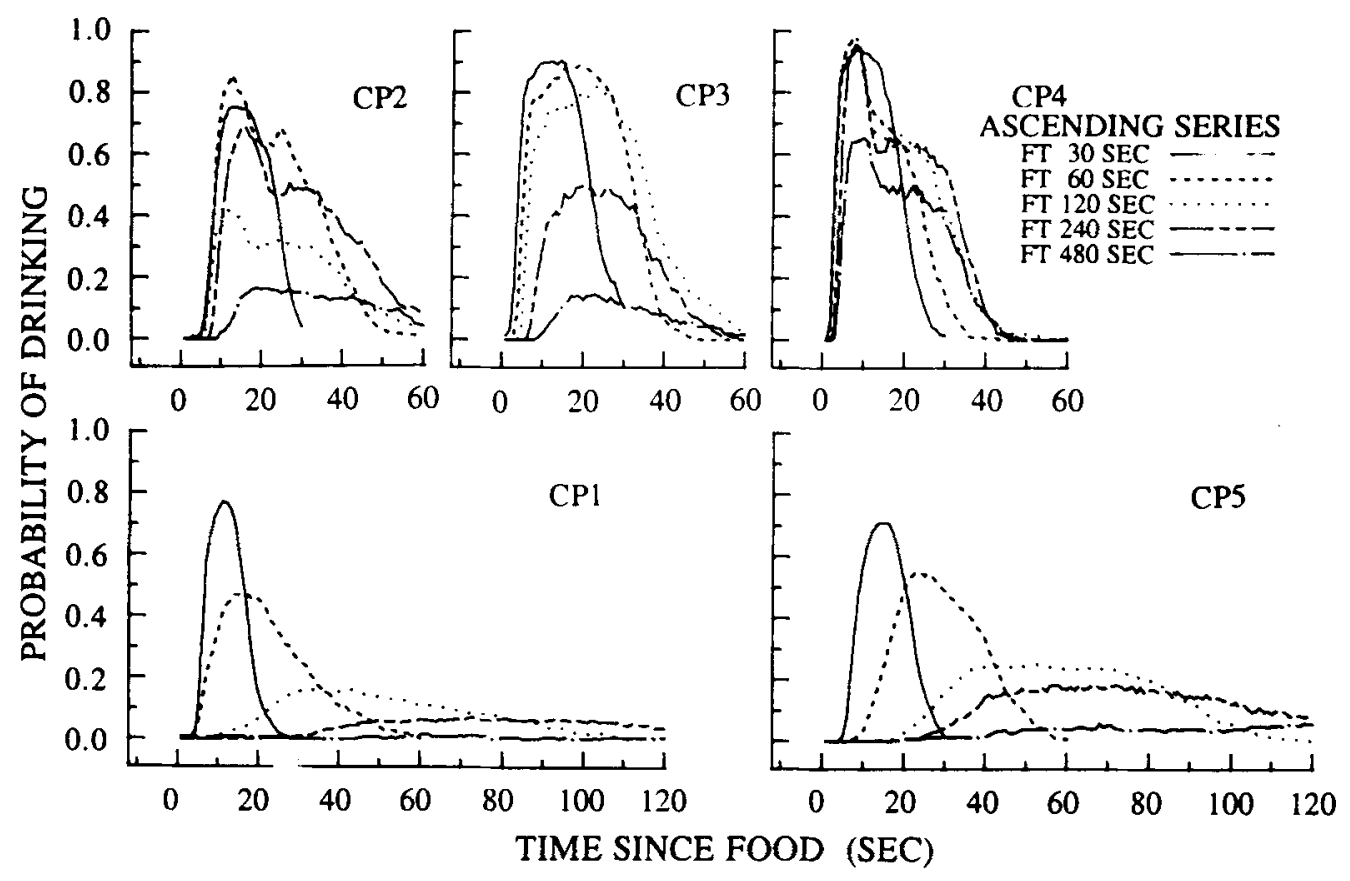

Figure 3. Temporal distribution of drinking within the interfood interval for each subject during the complete ascending series (Conditions 3-7, Table 1) of fixed-time (FT) schedules. These data are representative also of data from the first two conditions of the experiment (partial descending series, Conditions 1-2, Table 1); for brevity, data from these two conditions are omitted. The probability of drinking was computed by summing the number of $0.1-\sec$ bins containing drinking at time $t$ in each interfood interval across the last five sessions of a condition. This grand sum was then divided by the total number of 0.1 -sec periods at time $t$, to obtain an aggregate probability of drinking. Only the first 60 or $120 \mathrm{sec}$ of the interfood intervals are shown for the longer FT schedules; little or no drinking occurred in those portions of the interfood interval not displayed.

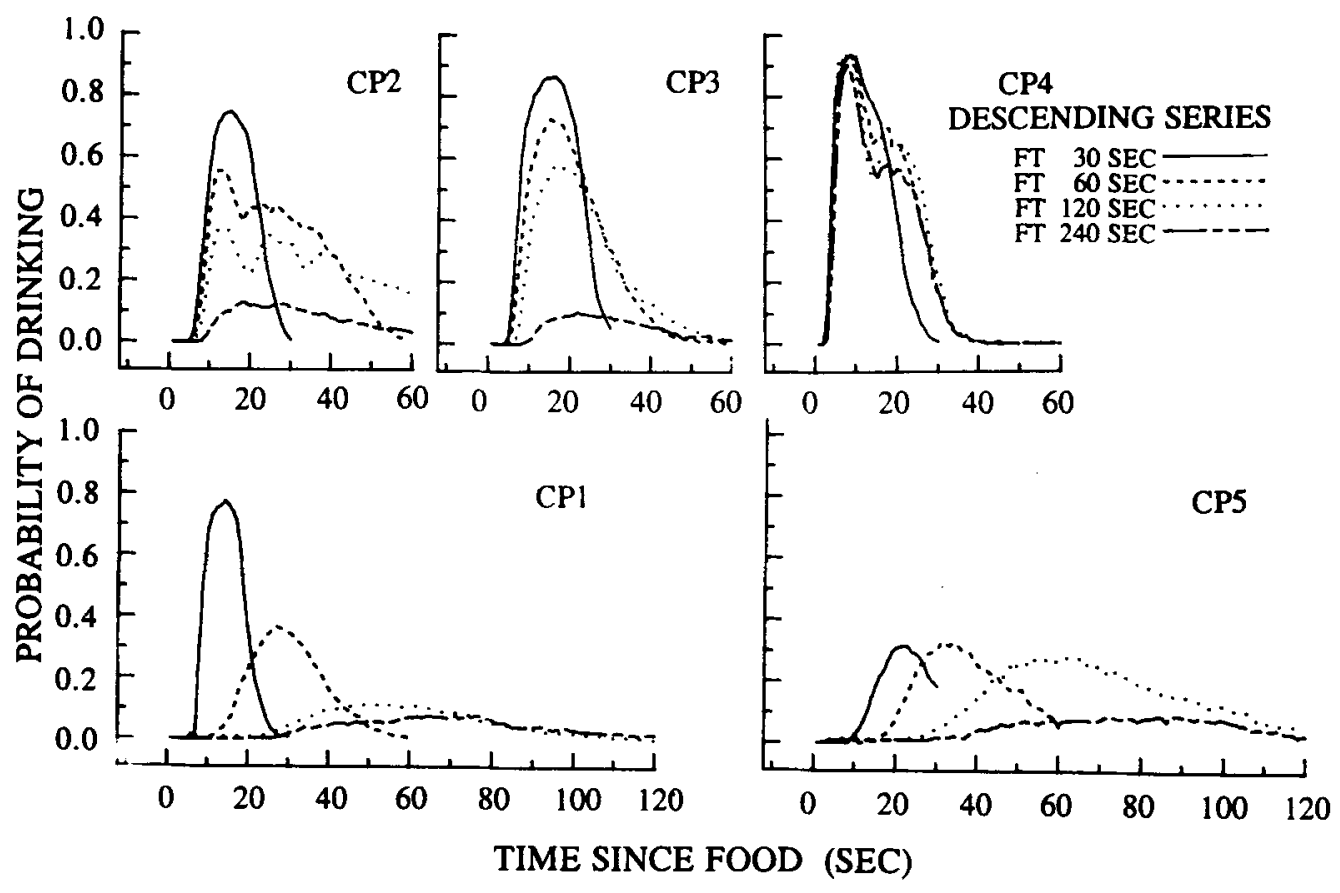

Figure 4. Temporal distribution of drinking within the interfood interval for each subject during the complete descending series (Conditions 8-11, Table 1) of fixed-time (FT) schedules. Only the first 60 or 120 sec of the interfood intervals are shown for the longer FT schedules; little or no drinking occurred in those portions of the interfood interval not displayed. Probability was computed as in Figure 3. 


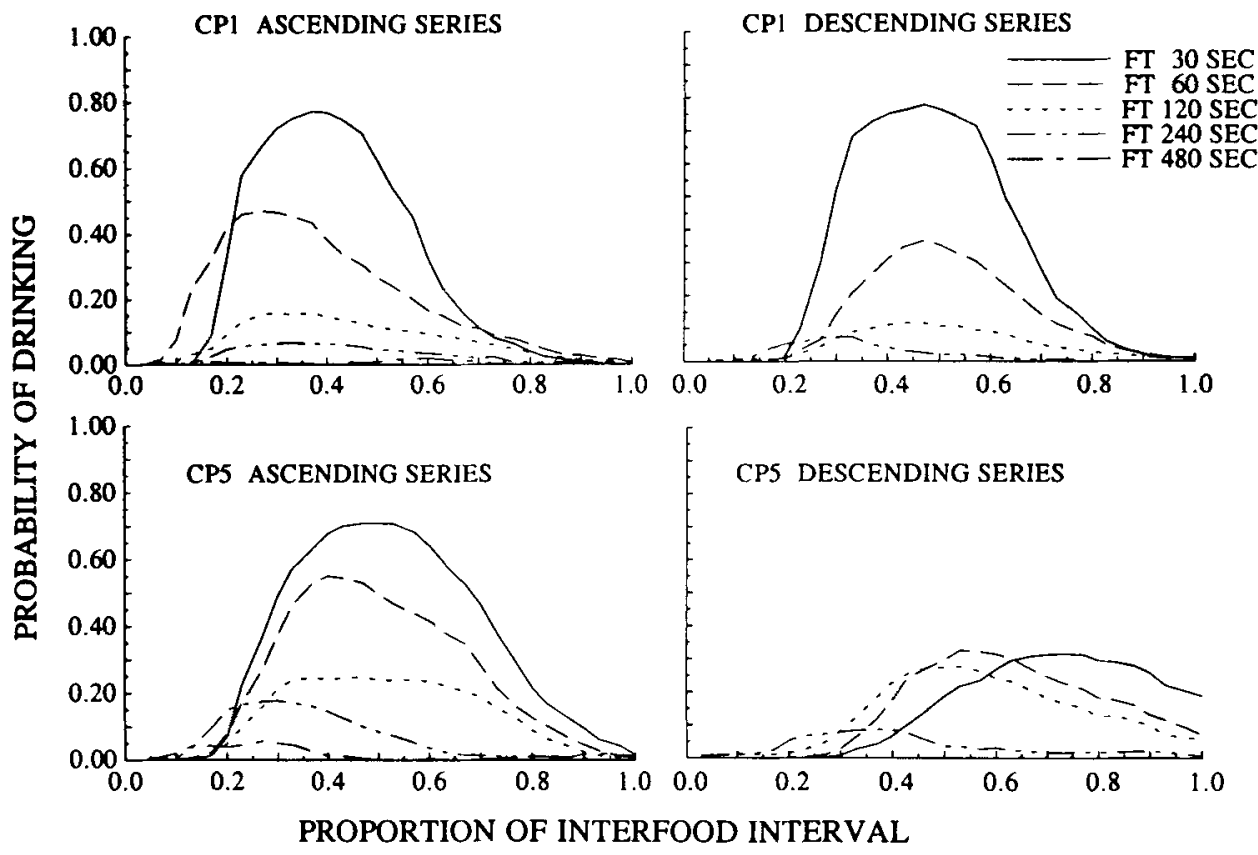

Figure 5. Temporal distribution of drinking within the interfood interval for Subjects CP1 and CP5 during the complete ascending (Conditions 3-7, Table 1) and descending (Conditions 8-11, Table 1) series of interfood intervals. The absolute time (in seconds) within the interfood interval for each condition was normalized by converting it to a proportion of the interfood interval; data were plotted as the amount of drinking versus the proportion of the interfood interval. Data have been transformed and replotted from Figures 3 and 4.

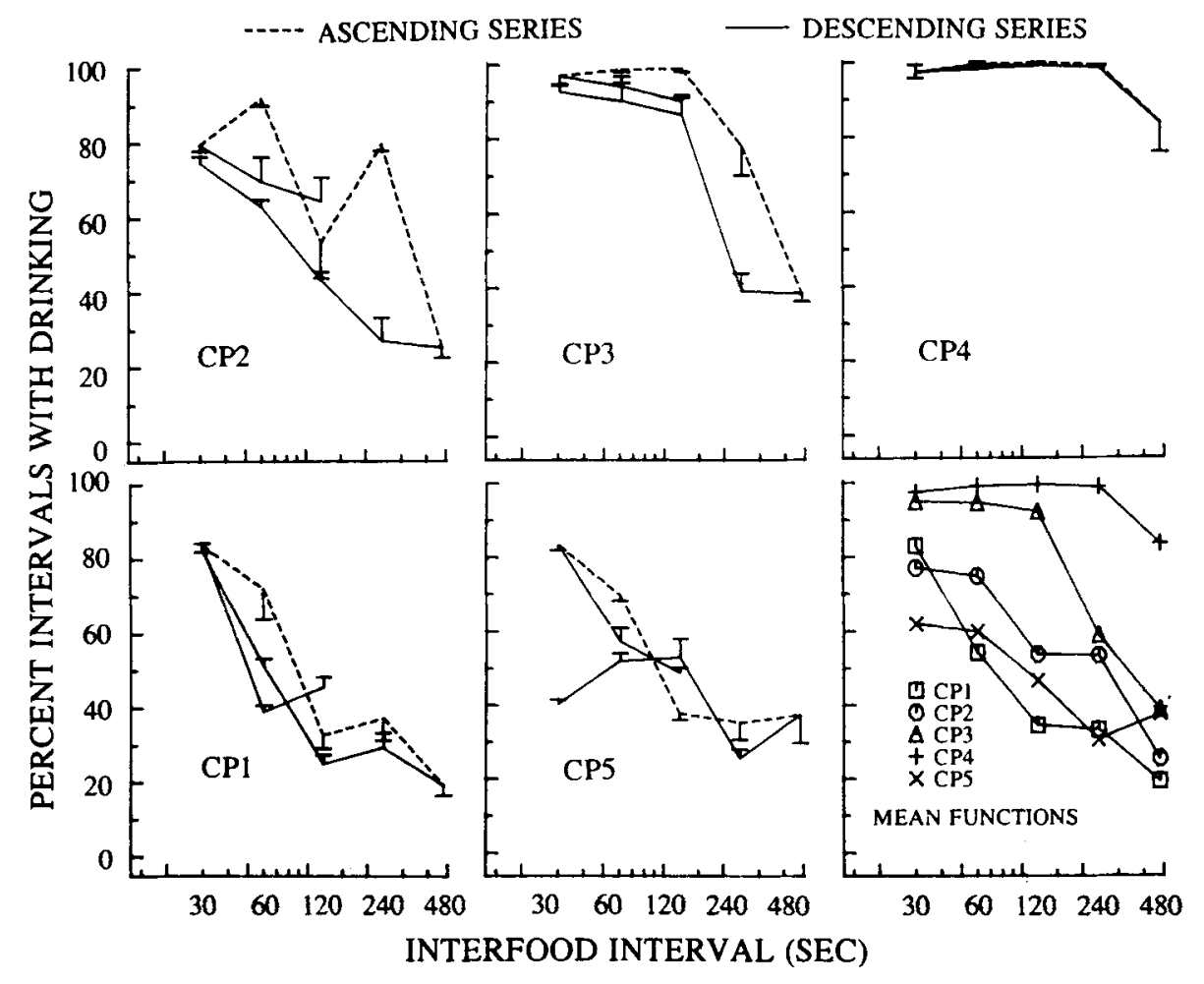

Figure 6. Percentage of intervals with drinking as a function of interfood interval duration for each subject. The abscissa is plotted in logarithmic coordinates to allow compact presentation of the data. Three separate functions are plotted on each graph. The two solid lines represent the partial (Conditions 1-2, Table 1) and complete (Conditions 8-11, Table 1) descending series of FT schedules. The dashed line represents data from the complete ascending series (Conditions 3-7, Table 1) of FT schedules. To make across-subject comparisons easier, each function plotted in the graph in the lower right corner represents the means of the ascending and descending functions for an individual subject. 
interval increased, although the function for CP4 was flat. There was a tendency for the 2 midinterval drinkers (CP1 and CP5) to initiate drinking bouts less frequently than the other subjects.

\section{Food-Tray Entry}

The temporal pattern of food-tray entry (the expected terminal behavior) was examined to determine whether subjects successfully discriminated each fixed-time schedule, as demonstrated by increased anticipatory food-tray entries near the end of the scheduled interfood interval.

Figures 7 and 8 show the probability of food-tray entry for each second within the interfood interval for each subject during the complete ascending (Figure 7) and descending (Figure 8) series of interval values. The overall patterns of food-tray entry were similar among subjects. Food-tray entry was high immediately after reinforcer delivery, then quickly declined and was low throughout the middle portion of the interval. It increased

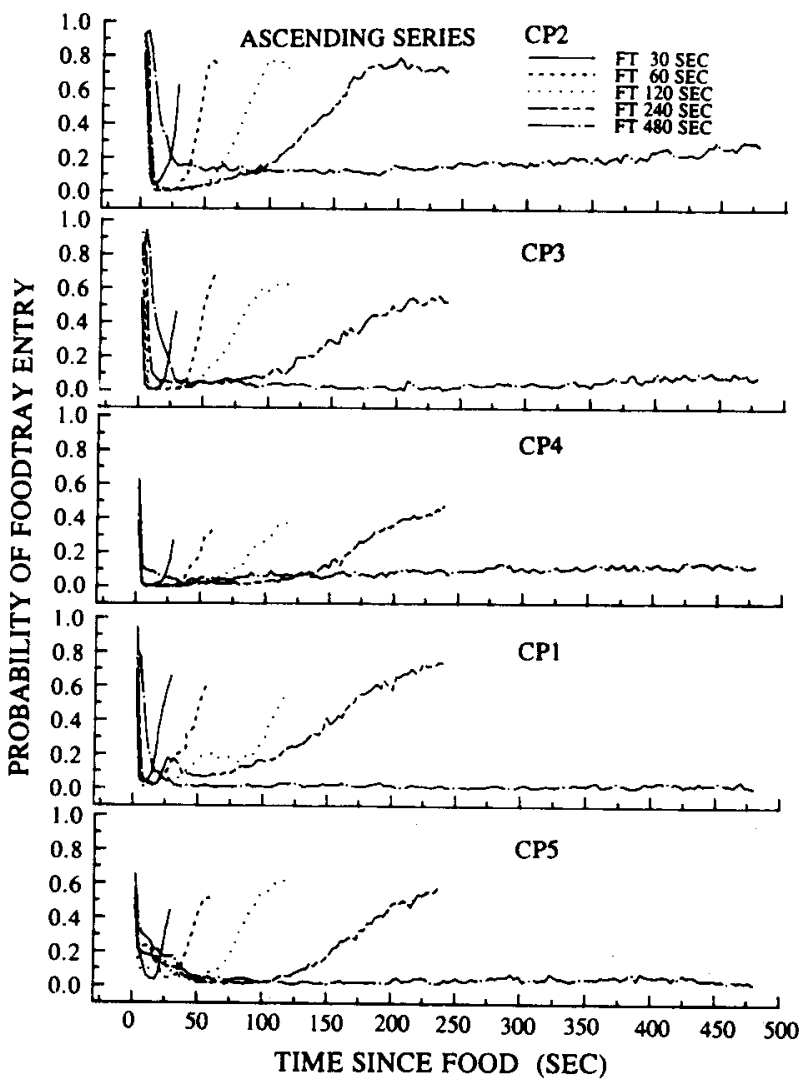

Figure 7. Temporal distribution of food-tray entry within the interfood interval for each subject during the complete ascending series (Conditions 3-7, Table 1) of fixed-time (FT) schedules. These data are also representative of data from the first two conditions of the experiment (partial descending series, Conditions 1-2, Table 1). For brevity, data from these first two conditions are omitted. The probability of food-tray entry was computed by summing the number of 0.1-sec bins containing food-tray entry at time $t$ in each interfood interval across the last five sessions of a condition. This grand sum was then divided by the total number of 0.1-sec periods at time $t$, to obtain an aggregate probability of food-tray entry.

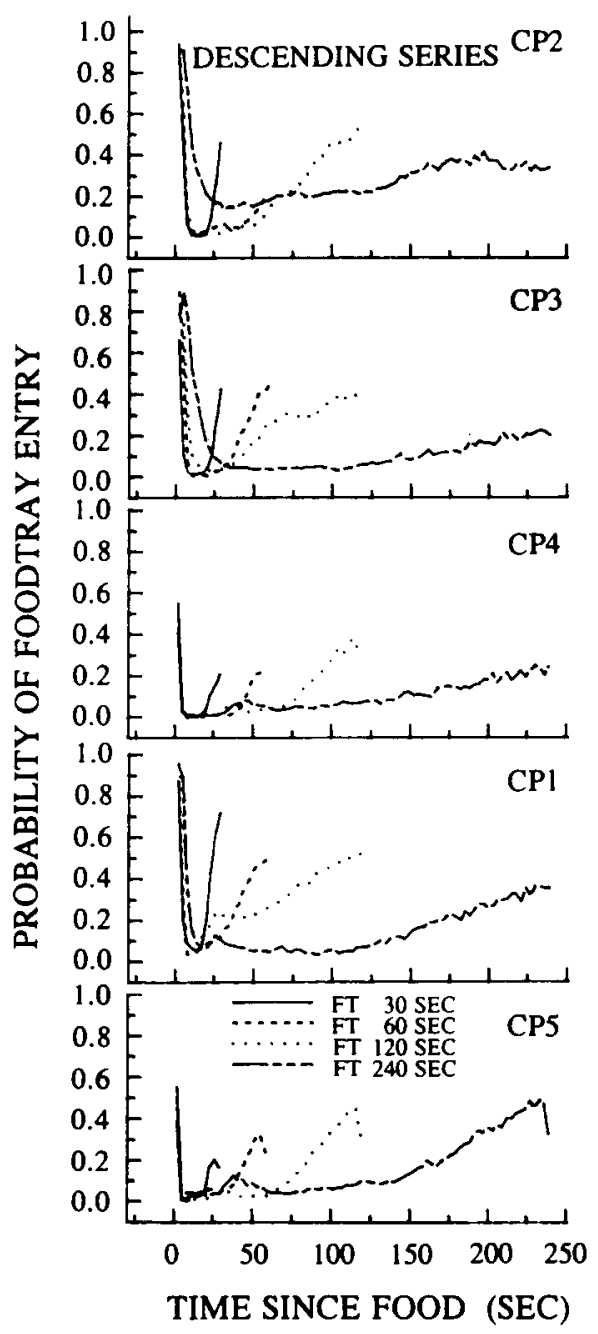

Figure 8. Temporal distribution of food-tray entry within the interfood interval for each subject during the complete descending series (Conditions 8-11, Table 1) of fixed-time (FT) schedules. Probability was computed as described in Figure 7.

steadily throughout the last half of the interval. In general, subjects remained at the food tray slightly longer following food delivery as the interfood interval increased. At the same time, food-tray entry rose more slowly at the end of the interval as the interval length increased from 30 to $240 \mathrm{sec}$, as evidenced by progressively shallower slopes of the food-tray functions. At the longest interval (FT $480 \mathrm{sec}$ ), Figure 7 shows that the food-tray functions decreased following reinforcer delivery and then remained at relatively low levels.

Quantification of food-tray entry temporal pattern. An index of curvature value (Fry, Kelleher, \& Cook, 1960) was computed for each of the probability functions for food-tray entry (as depicted in Figures 7 and 8 ) to provide a simple basis for comparing temporal discrimination between subjects and conditions. This index was computed for anticipatory food-tray entry during the last two thirds of the interfood interval; food-tray entry during the first 
third of the interfood interval was excluded because it included food-pellet retrieval and eating. The last two thirds of the interreinforcer interval was divided into 10 bins for each condition; thus, the index of curvature could vary from -.90 to +.90 . The best temporal regulation would be indicated by the positive maximum value of +.90 , meaning that food-tray entry occurred exclusively in the bin immediately preceding food delivery. Lower values would indicate less differential distribution of food-tray entry, with 0.0 indicating an even distribution of foodtray entry throughout the 10 bins.

Table 3 shows the index of curvature values for each subject and condition. In all cases, the index of curvature values were positive, indicating that some degree of temporal discrimination occurred in each condition. The highest values, indicating the best discrimination, were typically found at the short interfood intervals. In most cases, the lowest value for each subject occurred during the longest food schedule, FT $480 \mathrm{sec}$. A between-subject comparison showed that the index of curvature values were somewhat lower for CP1 and CP5 (the 2 midinterval drinkers) during the two shortest food schedules, FT 30 and FT $60 \mathrm{sec}$. In contrast, at interfood intervals greater than $60 \mathrm{sec}$, there was considerable overlap in the values for all subjects.

\section{Repetitive Pawgrooming}

We analyzed the temporal and nontemporal characteristics of pawgrooming for CP1 and CP5 to document this previously unreported adjunctive behavior and to compare it to drinking.

Temporal pattern of repetitive pawgrooming. Figure 9 shows the probability of pawgrooming within the interfood interval for CP1 and CP5 during the complete descending series (Conditions 8-11, Table 1) of interval values. Data from the longest interval value, FT $480 \mathrm{sec}$ (Condition 7, Table 1) are also shown. The pawgroom- ing distributions for CP1 and CP5 look very similar to the drinking distributions of the other subjects (Figures 3 and 4). Pawgrooming distributions were negatively skewed and sharply peaked; the maximum levels occurred shortly after food delivery (within the first $15 \mathrm{sec}$ ) at all interval lengths. As with the drinking distributions of the other 3 subjects, pawgrooming extended further into the interval at the longer FT schedules. We did not record the latency of pawgrooming; however, the overlapping peaks of the pawgrooming distributions in Figure 9 rule out the possibility that pawgrooming latency changed dramatically across conditions.

To facilitate comparisons between drinking and pawgrooming for CP1 and CP5, the probability distributions for both behaviors are plotted together in Figure 10 (CP1) and Figure 11 (CP5). For both subjects, the peak probability of pawgrooming occurred prior to that for drinking. There was some overlap in the distributions of drinking and pawgrooming at the shorter intervals, FT 30 and FT $60 \mathrm{sec}$. As the interval length increased, the distribution of drinking broadened and the amount of overlap decreased.

Frequency of pawgrooming. Figure 12 shows the percentage of intervals with pawgrooming as a function of the interfood interval for CP1 and CP5. Pawgrooming occurred in a high percentage of intervals for both subjects during each schedule condition, with the exception of a large decrease in frequency during the longest schedule, FT $480 \mathrm{sec}$, for CP1. A comparison of Figures 6 and 12 indicates that, in most cases, pawgrooming occurred with equal or greater frequency than drinking.

Time spent pawgrooming and drinking in schedule and baseline conditions. Figure 13 shows the percentage of session time spent pawgrooming for CP1 and CP5 during each schedule condition and the corresponding extinction baseline. In all cases, pawgrooming during extinction was reduced relative to the schedule condition.

Table 3

Index of Curvature Values for Food-Tray Entry

\begin{tabular}{|c|c|c|c|c|c|c|}
\hline \multirow[b]{2}{*}{ Condition } & \multirow{2}{*}{$\begin{array}{l}\text { Fixed-Time } \\
\text { Schedule } \\
\text { (sec) }\end{array}$} & \multicolumn{5}{|c|}{ Subject } \\
\hline & & CP1 & $\mathrm{CP} 2$ & CP3 & CP4 & CP5 \\
\hline 1 & 120 & 0.407 & 0.344 & 0.228 & 0.516 & 0.463 \\
\hline 2 & 60 & 0.386 & 0.534 & 0.495 & 0.576 & 0.292 \\
\hline 3 & 30 & 0.395 & 0.513 & 0.649 & 0.666 & 0.526 \\
\hline 4 & 60 & 0.373 & 0.562 & 0.627 & 0.593 & 0.470 \\
\hline 5 & 120 & 0.275 & 0.413 & 0.347 & 0.506 & 0.456 \\
\hline 6 & 240 & 0.305 & 0.271 & 0.326 & 0.474 & 0.472 \\
\hline 7 & 480 & 0.151 & 0.156 & 0.316 & 0.100 & 0.100 \\
\hline 8 & 240 & 0.383 & 0.120 & 0.320 & 0.295 & 0.405 \\
\hline 9 & 120 & 0.182 & 0.357 & 0.173 & 0.480 & 0.482 \\
\hline 10 & 60 & 0.327 & 0.384 & 0.481 & 0.597 & $*$ \\
\hline 11 & 30 & 0.504 & 0.719 & 0.664 & 0.607 & * \\
\hline
\end{tabular}

Note-Index of curvature values were calculated for food-tray entry during the last two thirds of the interfood interval in each condition. Ten time bins were used; thus, the index could vary from +0.9 to -0.9 . Calculations were performed acconding to the method outlined by Fry, Kelleher, and Cook (1960). *Index of curvature was not computed for this subject and condition because the changeover delay in effect resulted in occasional delays in food delivery; thus, the interfood interval was somewhat variable during this condition. Such variability makes comparisons across subjects and conditions ambiguous. 


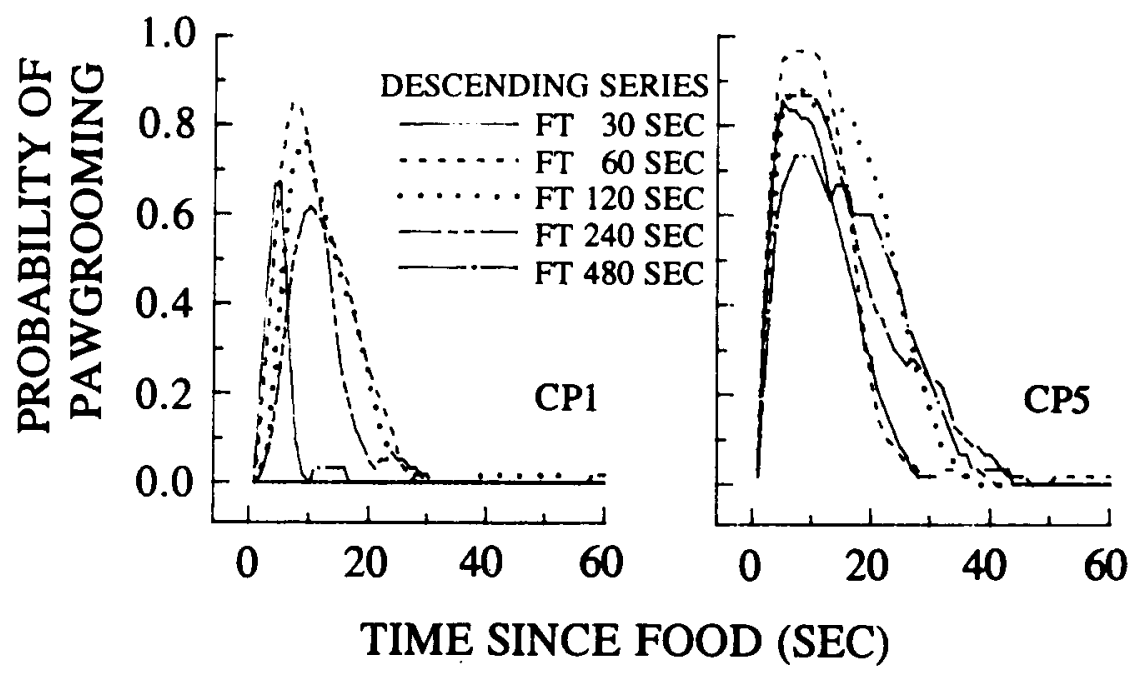

Figure 9. Temporal distributions of pawgrooming for CP1 and CP5 during the complete descending series (Conditions 8-11, Table 1) of fixed-time (FT) schedules. Data from the longest FT schedule, 480 sec (Condition 7, Table 1), are also shown. Data represent one complete session within the last five sessions of each condition. The probability of pawgrooming was computed for each second of the interfood interval by counting the number of interfood intervals containing pawgrooming at time $t$. This number was then divided by the total number of interfood intervals contained in that observation session to yield a probability measure.

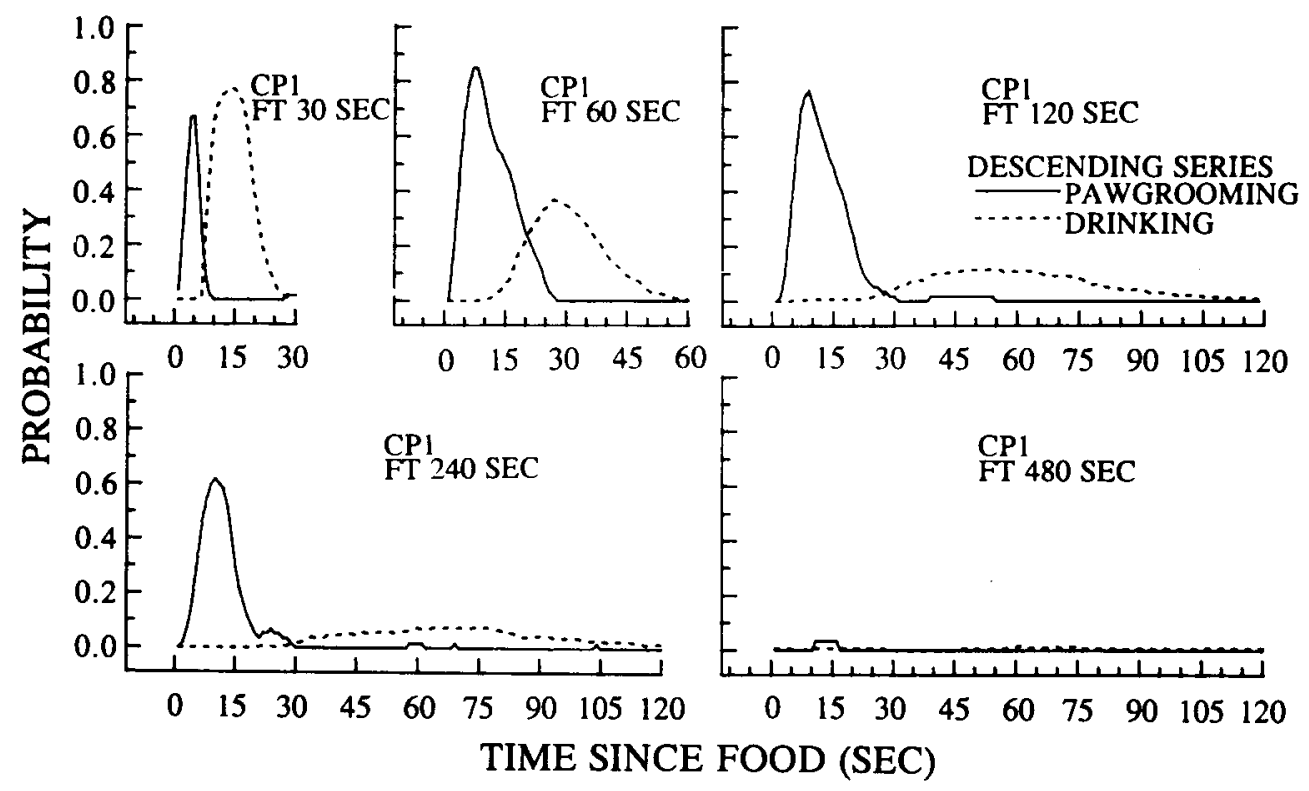

Figure 10. Temporal distributions of pawgrooming and drinking for Subject CP1 (Conditions 7-11, Table 1). Data are replotted from Figures 3, 4, and 9. 


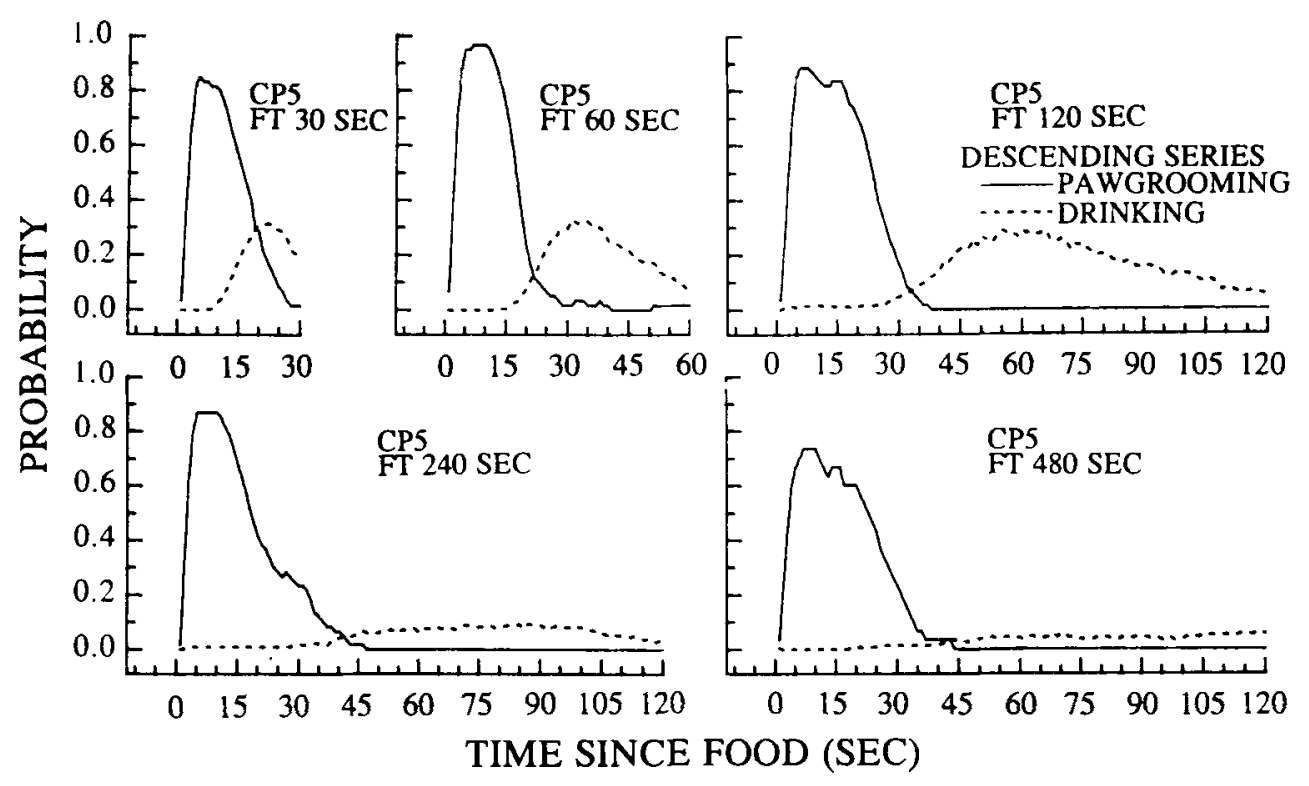

Figure 11. Temporal distributions of pawgrooming and drinking for Subject CP5 (Conditions 7-11, Table 1). Data are replotted from Figures 3, 4, and 9.

In this respect, pawgrooming was similar to drinking (Table 2). Figure 13 also shows that the percentage of session time spent pawgrooming generally increased as the interreinforcer interval length decreased. Figure 14 displays a similar pattern of increases in the percentage of session time spent drinking as the interreinforcer interval decreased for all subjects. Despite this overall similarity between subjects, there were some subtle between-subject differences in the percent of session time spent drinking. The 2 subjects who engaged in pawgrooming (CP1 and CP5) generally spent less time drinking than the other 3 subjects. A reexamination of Table 2 and Figure 6 reveals a corresponding tendency toward lower water intake and frequency of drinking for CP1 and CP5 compared to the other subjects.

\section{DISCUSSION}

\section{The Emergence of Two Distinct Patterns of Drinking}

Three subjects in this study (CP2, CP3, and CP4) initiated drinking shortly after each food delivery, over a

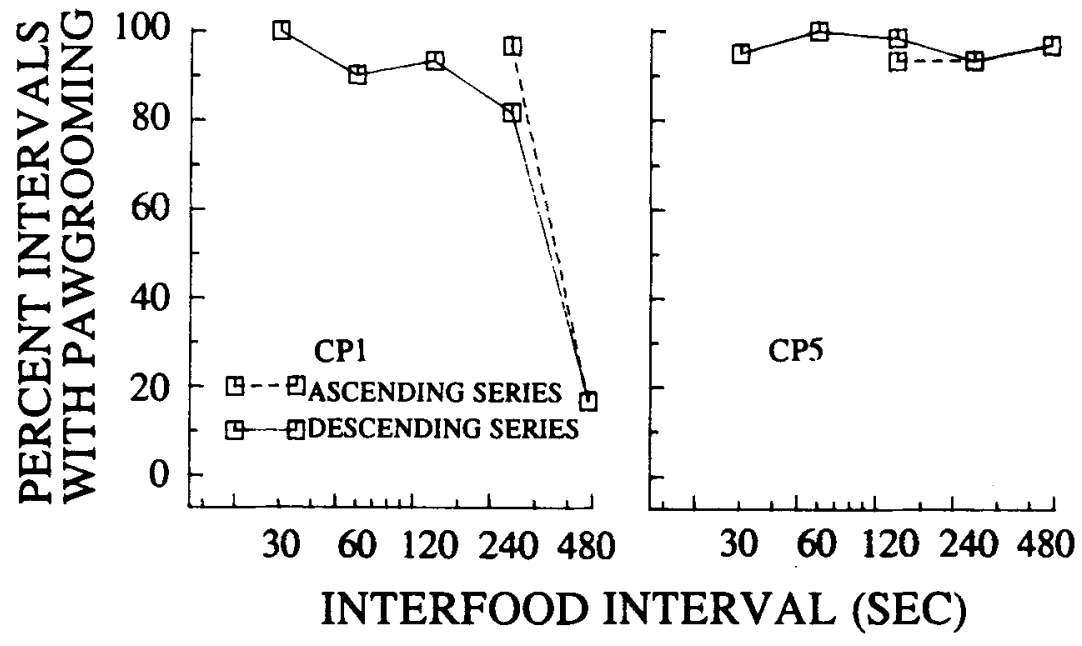

Figure 12. Percentage of intervals with pawgrooming for CP1 and CP5 as a function of interfood interval duration (in seconds). Data represent one complete session within the last five sessions of each condition (Conditions 7-11, Table 1). The abscissa is plotted in logarithmic coordinates to allow compact presentation of the data. 


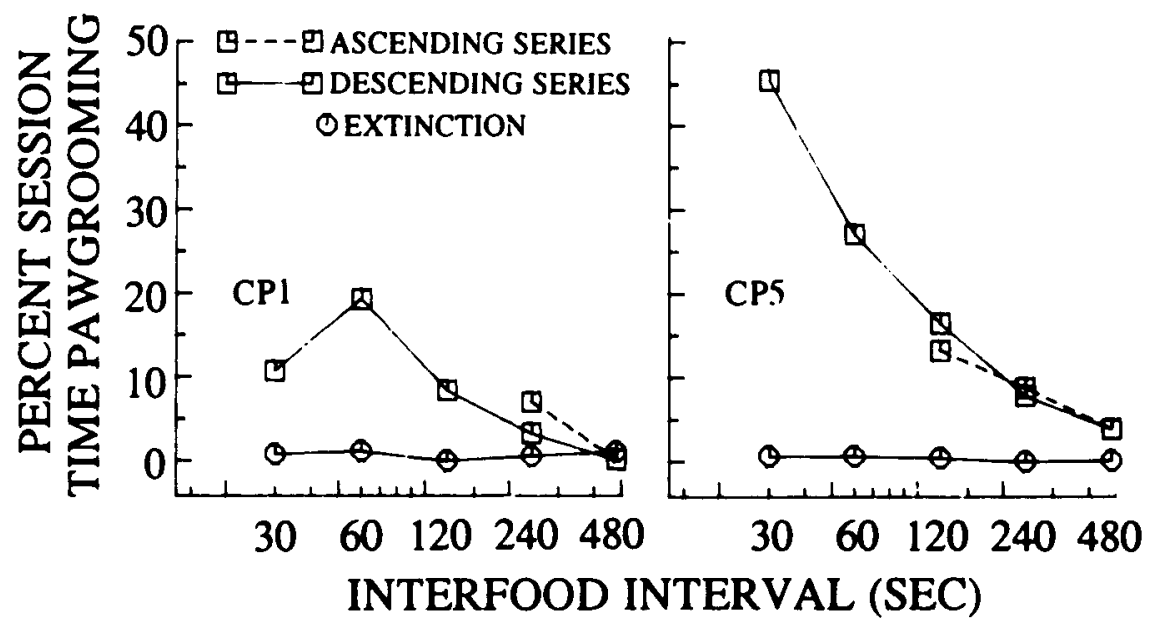

Figure 13. Percentage of session time spent pawgrooming for CP1 and CP5 as a function of interfood interval duration (in seconds). Data represent one complete session within the last five sessions of each condition (Conditions 7-11, Table 1). The percentage of session time spent pawgrooming during extinction sessions (EXT) is also shown. The abscissa is plotted in logarithmic coordinates to allow compact presentation of the data.

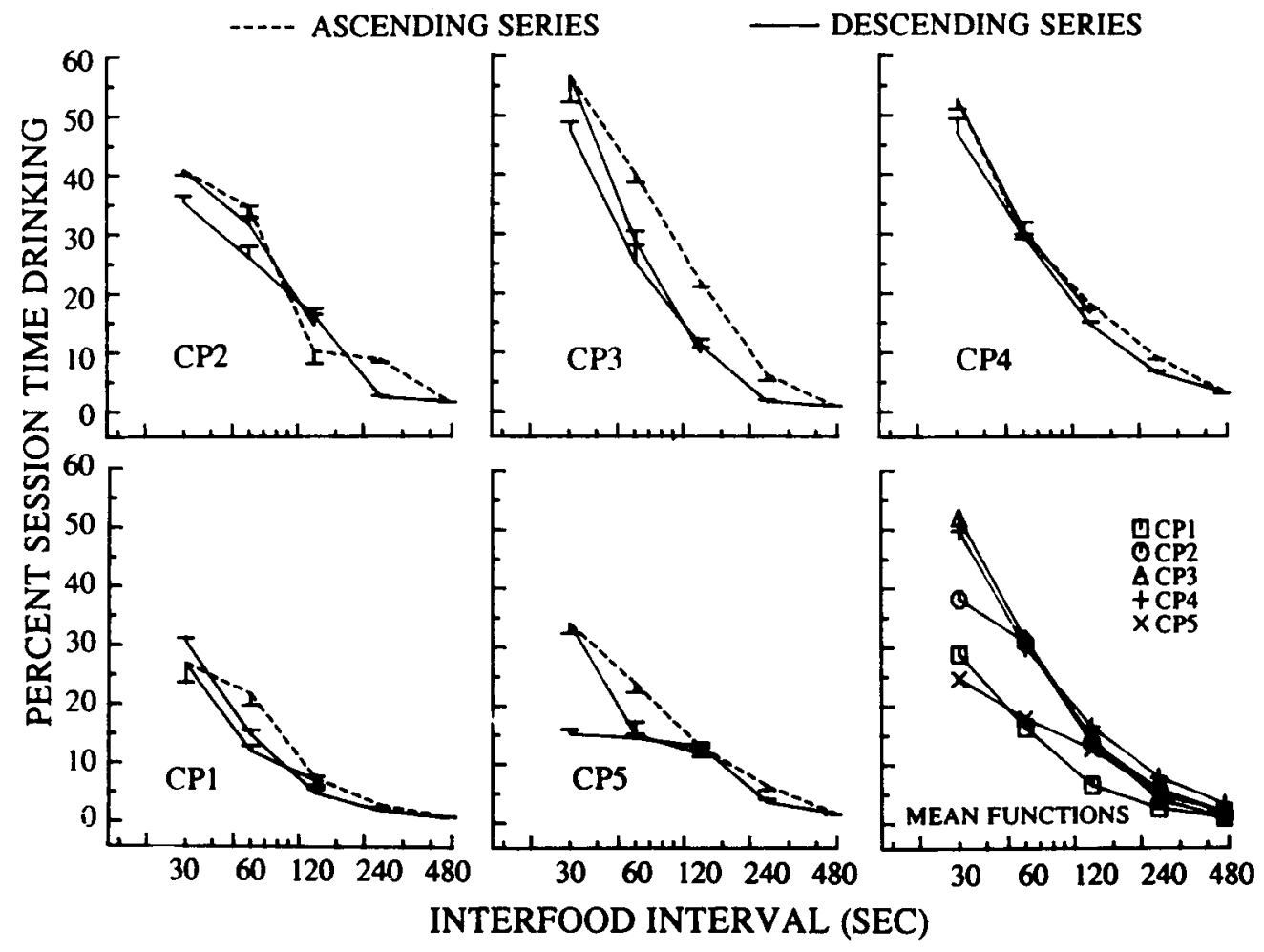

Figure 14. Percentage of session time spent drinking as a function of interfood interval duration (in seconds) for each subject and condition. The abscissa is plotted in logarithmic coordinates to allow compact presentation of the data. Three separate functions are plotted on each graph. The two solid lines represent the partial (Conditions 1-2, Table 1) and complete (Conditions 8-11, Table 1) descending series of FT schedules. The dashed line represents data from the complete ascending series (Conditions 3-7, Table 1) of FT schedules. To make across-subject comparisons easier, each function plotted in the graph in the lower right corner depicts the means of the ascending and descending series for an individual subject. 
wide range of food rates. The other 2 subjects (CP1 and CP5) showed a different and unexpected pattern; drinking was more variable, and usually occurred midinterval, such that peak levels of drinking showed large shifts to later times following food delivery as the interval length increased.

One possible explanation for the aberrant drinking patterns shown by CP1 and CP5 is that a gross deficiency in timing resulted in a failure of the food schedule to establish discriminative control over their behavior. Such a deficiency should have resulted in a failure of these subjects to anticipate food deliveries. The present study does not provide strong support for this possibility, because food-tray entry for both subjects increased consistently at the end of the scheduled interfood interval (Figures 7 and 8).

Although gross timing deficits can be ruled out, there was a tendency for CP1 and CP5, the 2 subjects who engaged both in drinking and in pawgrooming, to show less precise temporal regulation than the other 3 subjects (Table 3). In light of past speculation concerning the relation between the occurrence of adjunctive activities and the temporal regulation of operant behavior (e.g., Richelle \& Lejeune, 1980), it is tempting to suggest that the individual differences in the type and pattern of induced behavior seen in the present case were related causally to the observed differences in food-tray entry. An alternative possibility is that the tendency to engage in alternative forms of postfood adjunctive behavior (e.g., pawgrooming) and to display imprecise temporal discrimination reflect correlated individual differences among subjects. The study of such individual differences in behavior engendered by rewarding stimuli has generated increasing interest among behavioral neuroscientists (e.g., Mittleman \& Valenstein, 1984, 1985; Mittleman et al., 1988).

Could the unexpected midinterval drinking of CP1 and CP5 represent noninduced facultative behavior (Staddon, 1977)? Our data argue against this possibility because drinking was reduced in extinction and at long interfood intervals. The low probability of drinking in the last portion of the interfood interval suggests, furthermore, that drinking was not a terminal behavior (Staddon \& Simmelhag, 1971) for these 2 subjects. In light of these considerations, the drinking displayed by CP1 and CP5 appears to represent schedule-induced behavior. These data, along with other reports of induced drinking occurring at times other than the immediate postfood period (Rosellini \& Burdette, 1980; Segal et al., 1965), force the conclusion that induced drinking is not necessarily a postfood event.

\section{Pawgrooming}

Although the between-subject differences seen in drinking patterns were disconcerting initially, our subsequent finding that such differences predicted the occurrence of an unusual postreinforcer behavior, pawgrooming, was both exciting and provocative. Past research on grooming in adult rats indicates that pawgrooming is the initial segment of a stereotyped grooming bout; such pawgrooming is typically brief and progresses to nosewipes, ear- wipes, and general body grooming (Berridge, Fentress, \& Parr, 1987; Richmond \& Sachs, 1980). In contrast to this pattern, the rats in the present experiment engaged in pawgrooming for extended periods of time, and these bouts of repetitive pawgrooming were isolated from other forms of grooming. The finding that the percent of session time spent pawgrooming was reduced during extinction and at the longer interfood intervals suggests that pawgrooming was a schedule-induced behavior (Cohen \& Looney, 1984). This is surprising, inasmuch as previous research in the field of adjunctive behavior has concluded that grooming is not enhanced but rather suppressed by food-reinforcement schedules (e.g., Cook, et al., 1983; Roper, 1978), although in these studies, all forms of grooming were scored together in one category. The failure of such studies to find adjunctive grooming may be related to the indiscriminate scoring system employed. It is also possible that some unspecified feature of the present experimental space or paradigm was conducive to the development of postfood pawgrooming, rather than drinking, for some subjects.

\section{How Do These Results Bear on \\ Cohen et al.'s (1985) Two-State Model?}

According to the model, drinking should be a State I behavior, since it is a highly repetitive, stereotyped, species-specific activity. The model states that State I behaviors should occur immediately following food delivery. Drinking for three subjects occurred shortly after food delivery at all interfood interval durations, with only small increases in drink latency at the longer interfood intervals. These increases were more than 10 times smaller than those that would be expected if drinking accompanied State II; thus, drinking for these 3 subjects appears most consistent with the reactive State I pattern. Drinking patterns for the remaining 2 subjects (CP1 and CP5) were markedly different. Drink latency increased dramatically, in a roughly proportional manner (Figure 2), with each increase in interfood interval duration; furthermore, the drinking distributions appeared to be distributed with respect to relative time since food (Figure 5). Such a pattern most closely resembles the State II pattern, which is associated with gross locomotor and exploratory behaviors such as time-out responding and general activity.

We have suggested previously that drinking may accompany State II under some conditions if it becomes integrated with gross locomotor movements (Cohen et al., 1985). In such cases, we would expect drinking to occur in very short lick bursts, interspersed with other movements. Our observations in the present experiment, however, rule this out as an explanation for the drinking shown by CP1 and CP5 because we observed that instances of drinking occurred in uninterrupted bouts.

\section{Pawgrooming, Drinking, and \\ the Two-State Model}

Pawgrooming fits the criteria for a State I behavior; it is a repetitive, stereotyped activity and it occurs shortly after food delivery, regardless of the overall length of the 
interfood interval. Interestingly, pawgrooming is similar to drinking in several respects. First, pawgrooming occurs at the temporal locus usually associated with drinking. Second, the percentage of session time devoted both to drinking and to pawgrooming increases as the overall interval length decreases. Furthermore, like drinking for most subjects, pawgrooming is a high-probability behavior, particularly at short interfood interval lengths. In addition, both pawgrooming and drinking decrease dramatically during extinction sessions. Finally, the most obvious similarity is topographical; pawgrooming and drinking share a common motor component-licking. This latter similarity, together with the previous finding of schedule-induced air licking (Mendelson \& Chillag, 1970), strongly suggests that repetitive licking is a pervasive form of induced behavior in rats.

Given the many shared characteristics of pawgrooming and drinking, one intriguing possibility is that both are State I behaviors. If so, then the present experiment represents an interesting case, one in which some subjects allocated their time to more than one State I behavior. In its original form, the two-state model does not entertain this possibility explicitly. The occurrence of multiple State I behaviors would present particular problems if one relied solely on temporal criteria to assign a behavior to State I or State II. For instance, although the underlying motivational state may be a reactive process, only the first State I behavioral topography to occur would necessarily be reactive (i.e., peak at the same absolute time following reinforcer delivery). Any succeeding State I topographies would occur later in the interval, with their specific temporal loci partially dependent on the duration of the initial topographies.

In the present case, the purported State I behavior that occurred first following food delivery was pawgrooming. The delayed onset of drinking (another purported State I behavior) could be attributed directly to the preemptive occurrence of pawgrooming. An examination of Figures 10 and 11 suggests, however, that this simple explanation cannot fully account for the large increase in drink latency at the long interfood intervals. That is, the separation of the distributions of pawgrooming and drinking at long interval lengths suggests that other activities intervened between the two behaviors in such instances.

How might the two-state model account for a delay between pawgrooming and drinking in some conditions? Consider that if drinking and pawgrooming are State I behaviors, then both should serve a common function (i.e., modulation of physiological stress or arousal). The physiological consequences produced by engaging in the first State I behavior (i.e., pawgrooming) might be expected to reduce the motivation to engage in a second State I behavior (i.e., drinking). Drinking following pawgrooming could be viewed, thus, as a weak behavior that competed with other behaviors for expression. The result might be that other behaviors (e.g., facultative behaviors) occasionally intervened between drinking and pawgrooming. At short interfood intervals, such an attenuating effect of pawgrooming on drinking might be negligible because the initial induced motivation to engage in State I behaviors should be high (i.e., the two-state model proposes that the strength of State I increases as the interfood interval decreases). In contrast, at long interfood intervals, any pawgrooming-induced reduction in the motivation to drink might be significant because the initial induced motivation to drink is lower, whereas the strength of competing noninduced facultative behavior is presumably unchanged (Staddon, 1977). This hypothesized shift in the relative initial strength of State I behavior versus noninduced behaviors could produce the results observed in the present experiment-an increasing delay between pawgrooming and drinking as the interfood interval increased, and a lower frequency of drinking relative to pawgrooming (Figures 6 and 12) at long interfood intervals.

Although the above conjecture is post hoc and represents only one interpretation of the data, the implied interdependency of drinking and pawgrooming-the two proposed State I behaviors-leads to interesting and testable predictions. For instance, removing the opportunity to engage in one State I behavior should increase the duration of the second State I behavior and change its temporal locus. In contrast to an interdependency between multiple State I topographies, the two-state model would propose that multiple forms of State I behavior are independent of, and thus do not compete for expression with, State II behaviors. In the present case, if pawgrooming and drinking belonged to different states, then removing one should have had little effect on the other. Unfortunately, the advanced age of the subjects at the end of the experiment precluded manipulations of pawgrooming and drinking in the present experiment.

\section{Usefulness of Cohen et al.'s (1985) \\ Two-State Model}

The two-state model was successful, inasmuch as it predicted the occurrence of repetitive, stereotyped behavior following food delivery. The emergence of two repetitive postfood behaviors was not, however, anticipated. In its present form, the model does not provide a basis for predicting under what conditions multiple repetitive postfood behaviors will develop. In such cases, the possibility of two or more competing State I behaviors should be entertained; however, this means that temporal criteria may not be sufficient to predict whether a behavior belongs to State I or II. A straightforward extension of the model does predict a dependency between two behaviors within the same state, in contrast to the presumed independence of behaviors that belong to different states. Experimental manipulations of the two behaviors would be necessary to clarify the relation between the two behaviors in question. The status of pawgrooming and drinking in the present context remains unclear in the absence of such information.

In summary, our results demonstrate that the temporal locus of induced drinking can vary. We found that, for some subjects, drinking occurred immediately after food delivery, regardless of the overall length of the interreinforcer interval. For other subjects, drinking occurred 
midinterval, and an alternative form of lickingpawgrooming-occurred immediately postpellet. Perhaps past studies that reported drinking at times other than the immediate postreinforcer period (Rosellini \& Burdette, 1980; Segal et al., 1965) were also cases in which an alternative form of licking preceded drinking.

Our comparison of the characteristics of pawgrooming and drinking revealed many similarities. In particular, together with the previous reports of induced air-licking (e.g., Mendelson \& Chillag, 1970), the topographical similarity between pawgrooming and drinking suggests that intermittent reinforcement selectively enhances multiple forms of licking in the postfood period. Our results raise new questions about the development of multiple adjunctive behaviors and about their relationship to one another. Perhaps the most interesting concerns why one behavioral variation rather than another emerges as the dominant one, and why such variations have not been previously reported within the same experimental context.

\section{REFERENCES}

ATor, N. A. (1980). Mirror pecking and timeout under a multiple fixedratio schedule of food delivery. Journal of the Experimental Analysis of Behavior, 34, 319-328.

Azrin, N. H., Hutchinson, R. R., \& HAKe, D. F. (1966). Extinctioninduced aggression. Joumal of the Experimental Analysis of Behavior, 9, 191-204.

BerLyne, D. E. (1960). Conflict, arousal, and curiosity. New York: McGraw-Hill.

Berridge, K. C., Fentress, J. C., \& Parr, H. (1987). Natural syntax rules control action sequences in rats. Behavioural Brain Research, 23, 59-68.

Brantley, J. P. (1977). Effects of cues signalling a low probability of reinforcement on schedule-induced polydipsia. Unpublished master's thesis, University of Georgia.

BretT, L. P., \& Levine, S. (1979). Schedule-induced polydipsia suppresses pituitary-adrenal activity in rats. Journal of Comparative \& Physiological Psychology, 93, 946-956.

Brett, L. P., \& Levine, S. (1981). The pituitary-adrenal response to "minimized" schedule-induced drinking. Physiology \& Behavior, 26, 153-158.

Campagnoni, F. R., Lawler, C. P., \& Cohen, P. S. (1986). Temporal patterns of reinforcer-induced general activity and attack in pigeons. Physiology \& Behavior, 37, 577-582.

CANTOR, M. B. (1981). Bad habits: Models of induced ingestion in satiated rats and people. In S. Miller (Ed.), Behavior and nutrition (pp. 31-49). Franklin Institute Press.

Cohen, P. S., CAmpagnoni, F. R. (1989). The nature and determinants of spatial retreat in the pigeon between periodic grain presentations. Animal Learning and Behavior, 17, 39-48.

COHEN, P. S., \& LOONEY, T. A. (1984). Induction by reinforcer schedules. Journal of the Experimental Analysis of Behavior, 41, 345-353.

Cohen, P. S., Looney, T. A., Campagnoni, F. R., \& LaWler, C. P. (1985). A two-state model of reinforcer-induced motivation. In F. R. Brush \& J. B. Overmier (Eds.), Affect, conditioning and cognition: Essays on the determinants of behavior (pp. 281-297). Hillsdale, NJ: Erlbaum.

CoOK, P., Wallace, M. \& Singer, G. (1983). A reinterpretation of schedule-induced behaviors based on a systematic analysis of behavior. Neuroscience and Biobehavioral Reviews, 7, 97-104.

FALK, J. L. (1971). The nature and determinants of adjunctive behavior. Physiology \& Behavior, 6, 577-588.

FALK, J. L. (1981). The environmental generation of excessive behavior.
In S. J. Mule (Ed.), Behavior in excess: An examination of volitional disorders (pp. 313-337). New York: Free Press.

FRY, W., KeLLEhER, R. T., \& CoOK, L. (1960). A mathematical index of performance on fixed-interval schedules. Journal of the Experimental Analysis of Behavior, 3, 193-199.

Gibbon, J. (1977). Scalar expectancy theory and Weber's law in animal timing. Psychological Review, 84, 279-325.

Killeen, P. (1975). On the temporal control of behavior. Psychological Review, 82, 89-115.

Lucas, G. A., Timberlake, W., \& Gawley, D. J. (1988). Adjunctive behavior of the rat under periodic food delivery in a 24-hour environment. Animal Learning \& Behavior, 16, 19-30.

Mendelson, J., \& Chillag, D. (1970). Schedule-induced air licking in rats. Physiology \& Behavior, 5, 535-537.

Mittleman, G., Jones, G. H., Robins, T. W. (1988). The relationship between schedule-induced polydipsia and pituitary-adrenal activity: Pharmacological and behavioral manipulations. Behavioral Brain Research, 28, 315-324.

Mittleman, G., \&alenstein, E. S. (1984). Ingestive behavior evoked by hypothalamic stimulation and schedule-induced polydipsia are related. Science, 224, 415-417.

Mittleman, G., \& Valenstein, E. S. (1985). Individual differences in nonregulatory ingestive behavior and catecholamine systems. Brain Research, 348, 112-117.

Richelle, M. \& LEJEUNE, H. (1980). Time in animal behavior. New York: Pergamon.

Richmond, G., \& SACHS, B. D. (1980). Grooming in Norway rats: The development and adult expression of a complex motor pattern. Behaviour, 75, 82-96.

ROPER, T. J. (1978). The diversity and substitutability of adjunctive activities under fixed-interval schedules of food reinforcement. Journal of the Experimental Analysis of Behavior, 30, 83-96.

RoselLINI, R. A. (1985). An application of opponent-process theory to adjunctive behavior. In F. R. Brush \& J. B. Overmier (Eds.), $A f-$ fect, conditioning and cognition: Essays on the determinants of behavior (pp. 263-280). Hillsdale, NJ: Erlbaum.

Rosellini, R. A., \& BuRdeTte, D. R. (1980). Meal size and intermeal interval both regulate schedule-induced water intake in rats. $A n$ imal Learning \& Behavior, 8, 647-652.

SEGAL, E. F. (1969a). The interaction of psychogenic polydipsia with wheel running in rats. Psychonomic Science, 14, 141-144.

SEGAL, E. F. (1969b). Transformation of polydipsic drinking into operant drinking: A paradigm? Psychonomic Science, 16, 133-135.

SEgal, E. F., Oden, D. L., \& DeADWyler, S. A. (1965). Determinants of polydipsia: IV. Free reinforcement schedules. Psychonomic Science, 3, 11-12.

Shurtleff, D., Delamater, A. R., \& Riley, A. L. (1983). A reevaluation of the CS - hypothesis for schedule-induced polydipsia under intermittent schedules of pellet delivery. Animal Learning \& Behavior, 11, 247-254.

Staddon, J. E. R. (1977). Schedule-induced behavior. In W. K. Honig \& J. E. R. Staddon (Eds.), Handbook of operant behavior (pp. 125-152). Englewood Cliffs, NJ: Prentice-Hall.

STAdDon, J. E. R., \& Ayers, S. L. (1975). Sequential and temporal properties of behavior induced by periodic food deliveries. Behaviour, $54,26-49$.

Staddon, J. E. R., \&immelhag, V. L. (1971). The superstition experiment: A re-examination of its implications for the principles of adaptive behavior. Psychological Review, 78, 3-43.

WAYNER, M. J. (1970). Motor control functions of the lateral hypothalamus and adjunctive behavior. Physiology \& Behavior, 5, 1319-1325.

Wetherington, C. L., \& Riley, A. L. (1986). Schedule-induced polydipsia: Interactions with wheel nunning. Animal Learning \& Behavior, 14, 416-420.

(Manuscript received May 15, 1989; revision accepted for publication March 2, 1992.) 